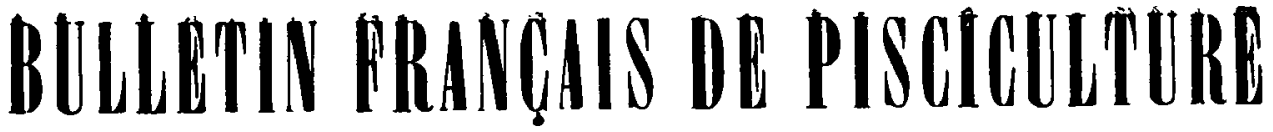

\section{QUELQUES MOTS SUR LE PLANCTON DES EAUX COURANTES}

\author{
Par M. le Professeur ANDRE \\ de l'Université de Genève.
}

Il y a quelque temps, un pisciculteur faisait appel à l'auteur de ces lignes pour être renseigné sur la capacité biogénique, - sur la valeur sitétique, si l'on préfère, - d'un bief long de quelques kilomètres, dans un tronçon duquel oṇ se proposait d'élever des alevins de truites fario jusqu'au stade de sommerling (1). Il s'agissait done de déterminer quelle quantité de nourriture se trouverait à la disposition des truites et, par conséquent, quel nombre approximatif on pourrait en élever dans ce bief avec quelques chances de succès. Le bief coulait parmi des taillis et le fond en était tapissé par des feuilles mortes, substratum peu propice au développement de la faune. Celle-ci, en effet, était assez pauvre; il y avait évidemment la nourriture exogène (insectes tombant des buissons bordant le bief), mais elle est difficile à évaluer et elle n'est guère utilisable par des alevins à cause de ses dimensions. Le résultat de l'examen du cours d'eau était donc plutôt défavorable. "Possible, disait le pisciculteur, mais il y a le plancton ". - "Eh! bien, non, cher Monsieur, répondis-je, il n'y a pas le jplancton $n$. - Dans les ruisseaux, exception faite pour quelques rares cas dont nous parlerons plus loin, le plancton est inexistant.

Cette notion erronée de l'existence du plancton dans les ruisseaux, je l'ai, non seulement entendue dans la bouche de pisciculteurs, mais trouvée aussi sous la plume de quelques auteurs et il est nécessaire d'en faire jus. tice, une fois pour toutes, espérons-le.

Il importe, tout d'abord, de donner une définition exacte du mot plancton. Celui-ci n'est pas, comme d'aucuns le croient volontiers, l'ensemble de la faune microscopique qui peuple toutes les eaux. Ce mol de plancton signifierait, en se basant sur l'étymologie greccue : crrant, tolalité des organismes errants; en réalité, son sens est plus restreint et il n'est applicable qu'à l'ensemble des organismes, végétaux et animaux, qui, sans se poser jamais sur le fond, passent toute leur existence nageant on flottanl,

(i) Truitelles d'un été. 
soil cutre deux caux, soit à la surface. Organısmes végétaux ou animaux, disions-nous ; en effet, on peut distinguer le phytoplancton (plancton végétal) et le zooplancton (plancton animal), vivant, bien entendu, intimement mélangés et plus ou moins dépendants l'un de l'autre, surtout au point de vue alimentaire. Le phytoplancton est constitué essentiellement de Bactéries, d'Algues microscopiques et de Flagellés ; directement il n'est pas utilisé par les Poissons, sauf peut-être par quelques alevins, mais il joue un rôle important dans l'alimentation du zooplancton. Dans les eaux douces, ce dernier est formé d'une façon presque exclusive de ćrustacés, de Rotateurs et de Protozoaires; ce sont les Crustacés qui tiennent la première place dans l'alimentation des Poissons.

Un peu de terminologie nous apprendra maintenant en quelques mots où vit le plancton : - haloplancton (plancton de la mer), - limnoplancton (des lacs), - héléoplancton (des étangs et des marais) - et potamoplancton (des fleuves). On remarquera qu'il n'existe aucun terme pour désigner le plancton des ruisseaux... et pour cause.

Laissons de côlé le plancton des étangs el des lacs ; l'importance de ce dernier pour la nourriture des poissons lacustres, tout particulièrement des Ombles et des Corégones, est trop connue pour que nous nous y attardions. Nous nous bornerons donc, comme l'indique le titre de ces lignes, aux eaux courantes.

Dans leur cours supérieur, les fleuves el les rivières sont assimilables : un ruisscau ou à un torrent et se montrent, comme nous le verrons plus loin, dépourvus de planclon. Pour ces cour's d'eau, au point de vue où nous nous plaçons maintenant, deux cas peuvent se présenter, qui d'ailleurs ne s'excluent pas, mais sont susceptibles au contraire de se combiner ensemble.

Dans l'un, le fleuve ou la rivière traverse un lac et alors l'émissaire entraîne à sa sortie une masse plus ou moins considérable de plancton lacustre. Par exemple le Rhône, à sa sortie du Léman, enlève annuellement à ce dernier, ainsi que nous l'avons calculé d'une façon pas trop approximative, 8.530 mètres cubes de plancton, ce qui fait à peu près le même nombre de tonnes, puisque la densité du plancton est sensiblement égale à celle dc l'eau. Encore ne s'agit-il là que du plancton qui peut être recueilli avec le filet en soie de bluterie à mailles très fines ; le nannoplancton, composé surtout de Bactéries et de Microflagellés, passe entre ces mailles et ne peut être récolté que par la méthode du centrifuge ( $\mathrm{I}$ ). En ce tronçon du cours d'eau la quantité de plancton est donc assez importante. Quelle sera la composition de ce limnoplancton devenu potamoplancton ? Elle sera, $\vec{a}$ peu de chose près, ce qu'elle était dans le lac, sauf que les planctontes de la profondeur seront absents ou plus ou moins rares selon l'état d'agitation du lac. Autre question : quel sera le sort de ce potamoplancton? Il disparaîtra graduellement, cela par la mort de ses composants, car les poissons fluviatiles ne sont guère planctophages, sauf peut-être à l'état d'alevins.

(1) E. André : - Sur le plancton du Rhône. - Revue d'Hydrologie, vol. III, rga6. 
Dans le second cas, nous voyons le fleuve, lorsque des pluies abondantes font déborder étangs et marais voisins, en recevoir des planctontes. Cela se présents surtout quand des bras morts avoisinent le fleuve ou la rivière : dans ces bras morts se développe un peuplement planctonique qui, lors des crues du cours d'eau, se répand dans ce dernier. Dans le potamoplancton, quelle qu'en soit l'origine, on peut encore constater la présence d'organismes végétaux ou animaux, vivant normalment sur le fond, qui en ont été détachés et qui ont été entraînés par le courant. Comme on le voit, le potamoplancton ne peut pas être considéré comme autochtone. Il est possible, cependant, que dans certains fleuves à courant extrêmement lent certains planctontes, notamment des phytoplanctontes, soient susceptibles de se multiplier.

Sur le cours inférieur de la Loire, dont le peuplement planctonique $t$ élé étudié par J. Des Cilleuls, cet auteur a noté que le phytoplancton prédomine beaucoup sur le zooplancton et que ce dernier est composé de queiques rares Héliozoaires, Rotateurs et Crustacés ; d'ailleurs aucun des composants du plancton de la Loire ne peut ètre considéré comme appartenant en propre au cours d'eau. Dans le produit recueilli par le filet fin, le plancton ne représente que la centième partic, le reste ćtant constituẻ par ce que l'on appelle le trypton, c'est à dire l'ensemble des débris microscopiques, d'origine animale, végétale ou minérale, se maintenant en suspension dans l'eau ( 1 ).

Revenons aux ruisseaux, qui dans le cas particulier, nous intéressent de façon plus spéciale. Si nous nous reportons à la définition du plancton, donnée plus haut, nous serons d'accord que celui-ci ne peut matériellement pas se développer dans un ruisseau. Des essais de peuplement de ruisseaux en plancton seraient toujours voués à l'insuccès, puisque toujours aussi les planctontes seraient emportés à l'aval avant d'avoir pu se reproduire. Cependant, il peut se produire en petit ce que nous constations pour les grands cours d'eau, c'est-à-dire que l'on peut parfois, mais très rarement, trouver des planctontes dans un ruisseau, lorsque des pluies abondantes ont fait déborder les étangs ou marais du voisinage, ou lorsque, cas exceptionnel, le ruisseau traverse un bassin d'eau stagnante ou y prend naissance. Notons aussi que, quelquefois, en plaçant dans un ruisseau un filet à plancton, on recueille ce que l'on nomme des tychoplanctontes, ou planctontes occasionnels, organismes du lit du cours d'eau qui, ayant imprudemment quitté leurs retraites, ont été emportés par le courant. Ces tychoplanctontes de ruisseau sont surtout des Gammarus (Crevettes d'eau douce) et diverses larves d'Insectes.

(I) J. DEs Custetzs. - Le phytoplancton de la Loire et de ses affluents dans la région saumuroise. - Internationale Revue der grsomten IIydrobiologic und Hydrographie Vol. XXI, igrg.

Nouvelle contribution à l'étude du phytoplancion de la Loire. - Ibid. Vol. XXVII, I 32 